\title{
AMENABILITY AND SUPERHARMONIC FUNCTIONS
}

\author{
S. NORTHSHIELD
}

(Communicated by J. Marshall Ash)

\begin{abstract}
Let $G$ be a countable group and $\mu$ a symmetric and aperiodic probability measure on $G$. We show that $G$ is amenable if and only if every positive superharmonic function is nearly constant on certain arbitrarily large subsets of $G$. We use this to show that if $G$ is amenable, then the Martin boundary of $G$ contains a fixed point. More generally, we show that $G$ is amenable if and only if each member of a certain family of $G$-spaces contains a fixed point.
\end{abstract}

Let $G$ be a countable group and $\mu$ a fixed probability measure on the subsets of $G$ such that $\mu$ is symmetric (i.e., $\mu(x)=\mu\left(x^{-1}\right)$ for all $x \in G$ ) and aperiodic (i.e., $\operatorname{supp}(\mu)$ contains a set of generators of $G$ ). Define $p(x, y)=$ $\mu\left(x^{-1} y\right)$. The function $p$ can be thought of as the transition matrix of a Markov chain $X$ with state space $G$. For $f$ a real function on $G$, define $p f(x)=\sum_{y \in G} p(x, y) f(y)$. We say that $f$ is harmonic or superharmonic iff $p f=f$ or $p f \leq f$ respectively.

By the representation theorem for positive harmonic functions, if the Martin boundary is trivial, then there exist no nonconstant positive harmonic functions. This of course implies that there are no nonconstant bounded harmonic functions on $G$ (in which case, we say that $G$ is Liouville). Is it true that if $G$ is Liouville, then $G$ has a trivial Martin boundary [A]? In an attempt to answer this, one could use the elegant proof that Liouville implies amenable [LS], and show that amenability implies trivial Martin boundary. This strategy is doomed, however, since the implication Liouville implies amenability is strict [KV].

Under the natural action of $G$ on the Martin boundary $M$ has at most one fixed point (see below). Although we cannot show that amenability implies that every point of $M$ is a fixed point (i.e., $M$ is trivial), we do show that amenability implies the existence of one fixed point in $M$. This will follow from Theorem 1, which states that $G$ is amenable if and only if every positive superharmonic function $f$ is nearly constant on arbitrarily large subsets of $G$. Although we are unable to prove that amenability is equivalent to the existence of a fixed point in the Martin boundary, we do show (Theorem 2) that

Received by the editors April 29, 1991 and, in revised form, February 26, 1992.

1991 Mathematics Subject Classification. Primary 43A07, 31C35; Secondary 31C05, $60 \mathrm{~J} 45$.

Key words and phrases. Amenable group, superharmonic function, Martin boundary, random walk. 
amenability is equivalent to the existence of a fixed point in each of a certain family of $G$-spaces, the Martin boundary being one.

The connection between superharmonic functions and amenability is based on the theorem of Kesten, which states that the spectrum of any symmetric and aperiodic probability has least upper bound 1 if and only if $G$ is amenable. We state a useful form of this theorem in the following lemma.

Lemma 1. $G$ is amenable iff $\inf \{\lambda: \exists f>0: p f \leq \lambda f\}=1$.

Proof. See [K, DK].

Theorem 1. $G$ is amenable iff for all positive superharmonic functions $f$ there exists a sequence $\left(x_{n}\right)$ in $G$ such that, for all $z, f\left(x_{n} z\right) / f\left(x_{n}\right) \rightarrow 1$ as $n \rightarrow \infty$.

Proof. Let $G$ be amenable and let $f$ be a positive superharmonic function. Define $p^{(n)}$ to be the $n$-fold convolution of $p$ with itself, and suppose that $\sup _{x} p^{(n)} f(x) / f(x) \neq 1$. Then there exists $\varepsilon \in(0,1)$ such that $p^{(n)} f \leq \varepsilon^{n} f$. Let $g=\sum_{0 \leq j \leq n-1} \rho^{(j)} f / \varepsilon^{j}$. Then $p g-\varepsilon g=p^{(n)} f / \varepsilon^{n-1}-\varepsilon f \leq 0$, which is, by Lemma 1 , a contradiction. Hence $\sup _{x} p^{(n)} f(x) / \tilde{f}(x)=1$.

For $x \in G$, let $f_{x}(z)=[f(x z) / f(x)]^{1 / 2}$. Note that

$$
\sum_{z} p(y, z) f(x z) / f(x)=\sum_{w} p(x y, w) f(w) / f(x) \leq f(x y) / f(x)
$$

and, therefore, $\left[f_{x}\right]^{2}$ is positive and superharmonic. Since $x \rightarrow x^{1 / 2}$ is concave and increasing, $f_{x}$ is also positive and superharmonic. Note that

$$
\begin{aligned}
p^{(n)} f_{x}(e) & =\sum_{z} p^{(n)}(e, z) f_{x}(z)=\sum_{z} p^{(n)}(e, z)[f(x z) / f(x)]^{1 / 2} \\
& =\sum_{y} p^{(n)}(x, y)[f(y) / f(x)]^{1 / 2}=p^{(n)}\left[f^{1 / 2}\right](x) / f(x)^{1 / 2}
\end{aligned}
$$

and, therefore, $\sup _{x} p^{(n)} f_{x}(e)=1$.

Since, for all $x, p^{(n)} f_{x}(e)$ is decreasing as a function of $n$, we can choose $x(k)$ such that, for all $n, p^{(n)} f_{x(k)}(e) \rightarrow 1$ as $k \rightarrow \infty$. We then have

$$
1=\left[f_{x(k)}(e)\right]^{2} \geq p^{(n)}\left[f_{x(k)}\right]^{2}(e) \geq\left[p^{(n)} f_{x(k)}(e)\right]^{2} \rightarrow 1 .
$$

Hence

$$
\sum_{z} p^{(n)}(e, z)\left[f_{x(k)}(z)-1\right]^{2}=p^{(n)}\left[f_{x(k)}\right]^{2}(e)-2 p^{(n)} f_{x(k)}(e)+1 \rightarrow 0 .
$$

By the aperiodicity of $\mu, f_{x(k)} \rightarrow 1$ pointwise.

Conversely, suppose that, for every positive superharmonic function $f$, there exists a sequence $x_{n}$ such that $f\left(x_{n} w\right) / f\left(x_{n}\right) \rightarrow 1$. Then

$$
p f\left(x_{n}\right) / f\left(x_{n}\right)=\sum_{z} p\left(x_{n}, z\right) f(z) / f\left(x_{n}\right)=\sum_{w} p(e, w) f\left(x_{n} w\right) / f\left(x_{n}\right) \rightarrow 1,
$$

so $\sup _{x} p f(x) / f(x)=1$. By Lemma $1, G$ is amenable.

For $x \in G$, let $x f$ denote the "left-translation" of $f$ by $x$ (i.e., $x f(y)=$ $f\left(x^{-1} y\right)$ ) and let $f_{x}(y)=x f(y) / x f(e)$ be the normalization of $x f$. We may then restate Theorem 1 . 
Corollary 1. $G$ is amenable if and only if, for all positive superharmonic functions $f$, the closure of the set $\left\{f_{x}: x \in G\right\}$ in the weak topology contains 1 (or, equivalently, the closure of the set $\{\lambda x f: \lambda>0, x \in G\}$ contains 1$)$.

Suppose Green's function $\Gamma(x, y)=\sum_{n>0} p^{(n)}(x, y)$ exists (equivalently, the random walk defined by $\mu$ is transient). Define the Martin function $K(x, y)$ $=\Gamma(x, y) / \Gamma(e, y)$. Since $p(z x, z y)=p(x, y)$, it follows that $\Gamma(z x, z y)=$ $\Gamma(x, y)$ and, therefore,

$$
K(x, z y)=K\left(z^{-1} x, y\right) / K\left(z^{-1}, y\right), \quad x, z \in G .
$$

By definition, the Martin compactification $M$ of $G$ corresponding to $\mu$ is the quotient of the set of sequences $y(n)$ in $G$ such that $K(\cdot y(n))$ converges pointwise by the relation where two sequences are equivalent if they give the same pointwise limits. The Martin function can then be extended. By (1), $z y(n)$ converges if and only if $y(n)$ converges and we may define an action of $G$ on $M$ by

$$
K(x, z \xi)=K\left(z^{-1} x, \xi\right) / K\left(z^{-1} \xi\right), \quad \xi \in M, x, z \in G .
$$

Corollary 2. If $G$ is amenable, then the Martin boundary has a unique fixed point.

Proof. If $\Gamma$ does not exist (equivalently, the random walk defined by $\mu$ is recurrent), then the Martin boundary is trivial.

Suppose that $\Gamma$ exists. By Theorem 1 , since $K(\cdot, y)=g_{y}$ where $g$ is the superharmonic function $\Gamma(\cdot, e)$, we see that $K(\cdot, \xi) \equiv 1$ for some $\xi \in M$. By equation (2), $\xi$ is a fixed point.

To show uniqueness, we note that for a fixed point $\xi$, (2) becomes

$$
K(x, \xi)=K\left(z^{-1} x, \xi\right) / K\left(z^{-1}, \xi\right)
$$

and, therefore, $K(\cdot, \xi)$ is a group homomorphism from $G$ to the positive real numbers. Since $K(\cdot, \xi)$ is superharmonic (because it is a limit of superharmonic functions), it follows by Lemma 2 below that $K(\cdot, \xi) \equiv 1$.

Lemma 2. All superharmonic homomorphisms from $G$ to the positive real numbers are trivial.

Proof. Let $F(x)$ be a superharmonic homomorphism from $G$ to the positive real numbers. Let $S$ be a maximal set with the property that, for each $x, S$ does not contain both $x$ and $x^{-1}$. Then

$$
\begin{aligned}
1 & =F(e) \quad(\text { since } F \text { is a homomorphism }) \\
& \geq p F(e) \quad(\text { since } F \text { is superharmonic }) \\
& =\sum_{x} \mu(x) F(x) \\
& =\sum_{x \in S} \mu(x)[F(x)+1 / F(x)] \quad \text { (since } F \text { is a homomorphism) } \\
& \geq 2 \mu(S) \quad(\text { since } F+1 / F \geq 2) \\
& =1 \quad(\text { since } \mu \text { is symmetric }) .
\end{aligned}
$$

Hence $\sum_{x \in S} \mu(x)[F(x)+1 / F(x)]=1$, so $F(x)=1$ for all $x$ in $S$ such that $\mu(x)>0$. Since $F$ is a homomorphism and since $G$ is generated by the support of $\mu$, we conclude that $F \equiv 1$. 
The converse of Corollary 2 is still open; however, Corollary 2 can be generalized. Recall that a $G$-space $M$ is a topological space $M$ together with an action $G \times M \rightarrow M$, which is continuous. This implies that, for all $g \in G$, the map defined by $x \rightarrow g x$ is continuous. Given a $G$-space $M, x \in M$ is called a fixed point if, for all $g \in G, g x=x$. Given a measure $\rho$ on $M$ and $g \in G$, we define a measure $\rho^{g}$ by $\rho^{g}(A) \equiv \rho\left(g^{-1} A\right)$.

Definition. A $(G, \mu)$-superharmonic measure space $(M, \rho)$ is a $G$-space $M$ with a measure $\rho$ on the Borel sets of $M$ such that

(a) $M$ is locally compact,

(b) $\rho(A)>0$ for all nonempty open sets $A$ (i.e., $\operatorname{supp}(\rho)=M$ ),

(c) $\sum_{g} \mu(g) \rho^{g}(A) \leq \rho(A)$ for all Borel sets $A$,

(d) $\rho^{g} \ll \rho$ for all $g \in G$, and

(e) $d \rho^{g} / d \rho$ is continuous on $M$.

Lemma 3. For $g, h \in G$ and $\rho$-almost all $x \in M$,

(a) $d \rho^{g h} / d \rho^{g}(x)=d \rho^{h} / d \rho\left(g^{-1} x\right)$, and

(b) $f(g)=\left[d \rho^{g} / d \rho\right](x)$ is superharmonic.

Proof. (a) For any Borel subset $A$ of $M$,

$$
\begin{aligned}
\int_{A}\left[d \rho^{g h} / d \rho^{g}\right](x) d \rho^{g}(x)=\rho^{g h}(A) & =\rho^{h}\left(g^{-1} A\right) \\
=\int g^{-1} A\left[d \rho^{h} / d \rho\right](x) d \rho(x) & =\int_{A}\left[d \rho^{h} / d \rho\right]\left(g^{-1} x\right) d \rho^{g}(x) .
\end{aligned}
$$

(b) For any Borel subset $A$ of $M$,

$$
\begin{aligned}
& \int_{A} \sum_{h} \rho(g, h)\left[d \rho^{h} / d \rho\right](x) d \rho(x) \\
& \quad=\sum_{h} p(g, h) \rho^{h}(A)=\sum_{h} \mu(h) \rho^{h}\left(g^{-1} A\right) \\
& \quad \leq \rho\left(g^{-1} A\right)=\int_{A}\left[d \rho^{g} / d \rho\right](x) d \rho(x) .
\end{aligned}
$$

Let $M^{*}$ be the unique compactification of $M$ such that, for every $g \in G$, the function $d \rho^{g} / d \rho$ can be extended to a continuous function on $M^{*}$ and the family of such extended functions separates $\partial M \equiv M^{*} \backslash M$ (see [B]). We call $M^{*}$ the natural compactification of $M$.

This definition is motivated by the following example. Assume that Green's function $\Gamma$ exists. For $A \subset G$, let $\rho(A)=\sum_{x \in A} \Gamma(x, e)$ and let $M=G$ where $G$ acts on $M$ by multiplication on the right. Then $(M, \rho)$ is a $(G, \mu)$ superharmonic measure space and the natural compactification $M^{*}$ of $M$ is the Martin compactification of $G$ (see [B]).

We now extend the action of $G$ on $M$ to an action of $G$ on $M^{*}$. Let $x_{n}$ and $y_{n}$ be sequences in $M$, which both converge to $\xi \in M^{*}$. Suppose that the sequences $g x_{n}$ and $g y_{n}$ converge to $\xi_{1}$ and $\xi_{2}$ respectively. We now show that $\xi_{1}=\xi_{2}$. By condition (b) above, $\rho$-null sets have empty interior and, therefore, without loss of generality, we assume $x_{n}, y_{n}$ satisfy Lemma 2 . 
Then, for $h \in G$,

$$
\begin{aligned}
d \rho^{h} / d \rho\left(\xi_{1}\right) & =\lim _{n \rightarrow \infty}\left[d \rho^{h} / d \rho\right]\left(g^{-1} x_{n}\right) \quad(\text { by }(\mathrm{e})) \\
& =\lim _{n \rightarrow \infty}\left[d \rho^{g h} / d \rho^{g}\right]\left(x_{n}\right) \quad(\text { by Lemma 3(a)) } \\
& =\left[d \rho^{g h} / d \rho^{g}\right](\xi) \\
& =\left[d \rho^{h} / d \rho\right]\left(\xi_{2}\right) \quad \text { (as above). }
\end{aligned}
$$

Since the functions $d \rho^{g} / d \rho$ separate points, we have $\xi_{1}=\xi_{2}$. Hence we may define an action of $g$ on $M^{*}$ by

$$
g \xi=\lim _{n \rightarrow \infty} g x_{n}
$$

where $x_{n}$ is any sequence in $M$, which converges to $\xi$.

Theorem 2. $G$ is amenable iff for any $(G, \mu)$-superharmonic measure space $(M, \nu)$ the natural compactification of $M$ has a fixed point.

Proof. Let $G$ be amenable. By Lemma 3 and Theorem 1 , there exists $x \in M$ such that there exists a sequence $g(n)$ in $G$ such that

$$
\left[d \rho^{k} / d \rho\right]\left(g(n)^{-1} x\right)=\left[d \rho^{g(n) k} / d \rho^{g(n)}\right](x) \rightarrow 1 \text { as } n \rightarrow \infty .
$$

Since $M^{*}$ is sequentially compact (because $\partial M$ is metrizable), we assume $x_{n} \equiv g(n)^{-1} x$ converges to, say, $\xi$. Then $\left[d \rho^{k} / d \rho\right](\xi)=1$ for all $k \in G$. Hence, for all $k$,

$$
\left[d \rho^{k} / d \rho\right]\left(g^{-1} \xi\right)=\left[d \rho^{g k} / d \rho\right](\xi) /\left[d \rho^{g} / d \rho\right](\xi)=1=\left[d \rho^{k} / d \rho\right](\xi) .
$$

Therefore, for all $g \in G, g \xi=\xi$.

Suppose that for every $(G, \mu)$-superharmonic measure space $(M, \nu), M^{*}$ has a fixed point. Let $f$ be a superharmonic function, $M=G$, and, for $A \subset M, \rho(A)=\sum_{g \in A} f(g)$. Also, let $G$ act on $M$ by right multiplication. By the symmetry of $\mu,(M, \rho)$ is a $(G, \mu)$-superharmonic measure space.

Let $\xi$ be a fixed point in $M^{*}$. Note that since $F \equiv\left[d \rho^{*} / d \rho\right](\xi)$ is a limit of superharmonic functions, it is superharmonic. Also,

$$
\begin{aligned}
F(h g) / F(h) & =\left[d \rho^{h g} / d \rho^{h}\right](\xi) \\
& =\lim _{n \rightarrow \infty}\left[d \rho^{h g} / d \rho^{h}\right]\left(x_{n}\right)=\lim _{n \rightarrow \infty}\left[d \rho^{g} / d \rho\right]\left(x_{n} h^{-1}\right) \\
& =\left[d \rho^{g} / d \rho\right]\left(h^{-1} \xi\right)=F(g) .
\end{aligned}
$$

Therefore, $F$ is a homomorphism from $G$ to the multiplicative group of positive real numbers. By Lemma $2, F \equiv 1$.

Let $g_{n} \rightarrow \xi$. Then, for all $h \in G$,

$$
\lim _{n \rightarrow \infty} f\left(g_{n} h\right) / f\left(g_{n}\right)=\lim _{n \rightarrow \infty}\left[d \rho^{h} / d \rho\right]\left(g_{n}\right)=1 \text {. }
$$

Since $f$ was arbitrary, $G$ is amenable by Theorem 1 .

\section{ADDED IN PROOF}

Ph. Bougerol and L. Elie have shown (personal communication) that a discrete Liouville group need not have trivial Martin boundary.

\section{ACKNOWLEDGMENT}

The author thanks the referee for several useful suggestions. 


\section{REFERENCES}

[A] David Aldous, personal communication.

[B] M. Brelot, On topologies and boundaries in potential theory, Lecture Notes in Math., vol. 175, Springer, New York, 1971.

[DK] Joseph Dodziuk and Leon Karp, Spectral and function theory for combinatorial Laplacians, Geometry of Random Motion, Contemp. Math., vol. 73, Amer. Math. Soc., Providence, RI, 1988, pp. 25-40.

[K] H. Kesten, Full Banach mean values on countable groups, Math. Scand. 7 (1959), 146-156.

[KV] V. A. Kaimanovich and A. M. Vershik, Random walks on discrete groups: boundary and entropy, Ann. Probab. 11 (1983), 457-490.

[LS] T. J. Lyons and D. Sullivan, Function theory, random paths and covering spaces, J. Differential Geom. 19 (1984), 299-323.

Department of Mathematics, State University of New York College at Plattsburgh, Plattsburgh, New York 12901

E-mail address: northssw@splava.cc.plattsburgh.edu 